\section{Value of Heart Rate Decelera- tion Capacity in Evaluation of Autonomic Nervous Functions in Type 2 Diabetes}

\section{Sir,}

Autonomic neuropathy of diabetes is a kind of neuropathy caused by diabetic microangiopathy and abnormal glucolipid metabolism, and is also one of the common complications in patients with diabetes. ${ }^{1}$ Its clinical manifestations are complicated, and its onset is hidden. In addition, it is mostly irreversible and seriously affects patients' prognosis. Although autonomic neuropathy of diabetes received attention early, its onset is very hidden, and it is difficult to diagnose it in the early stage. It was diagnosed by traditional cardiovascular reflex test in the past, but the sensibility is very low, and there is lack of uniform diagnostic criteria. ${ }^{2}$ The value of heart rate deceleration capacity (DC) can be detected under physiological conditions, free from the impacts of multiple external factors such as circadian rhythms, body temperature, routine activities and premature beat. It has the advantages of independentand quantitative determination of vagus nerve regulation function, and has better specificity and sensitivity. ${ }^{3}$ The author used DC to evaluate autonomic nervous functions of patients with type 2 diabetes and also found the high diagnostic value of DC.

Eighty-three patients with type 2 diabetes, who were received and treated in Chinese PLA General Hospital, PLA Medical College, from February 2017 to August 2019, were chosen in Group A. The study was approved by the Hospital's Ethics Committee. Inclusion criteria of diabetes patients were the diagnostic criteria of type 2 diabetes. Patients with hypertension, myocardial infarction, cardiac insufficiency, hyperthyroidism and other diseases affecting heart rate and / or autonomic nerve disease were excluded. Sixty-five healthy persons, who underwent the physical examination in the same period, were chosen in Group B. The criteria of healthy people included absence of any subjectivesymptom, and chronic disease history, with negative urine glucose, normal blood glucose; no abnormality in routine electrocardiogram, ultrasound cardiogram and chest $X$ film; no myocardial ischemia evidence and arrhythmia in 24 hours long-range electrocardiogram monitoring.

All foods and medicines affecting cardiac autonomic nerve functions were forbidden one day before the examination for both Group A and Group B, including strong tea, coffee, and wine. Both groups worked and rested according to the rules, avoided strenuous exercise and rage and ensured sleep.

DC test was conducted for both groups; namely, 24 hours dynamicelectrocardiography wasconducted, and DC wascalculated offline. The specific calculation method of DC was that the original electrocardiogram information from 24 hours ambula- tory electrocardiogram was screened with the $500 \mathrm{~Hz}$ digital automatic processing system to select the deceleration cycles; and the heart rate value was fixed as 20 cycles. The deceleration point of heartbeat intervals longer than the preceding interval was selected as the centre point. The heart rate segments were arranged in order and phased. Then the averages of the corresponding periods were calculated respectively. $X(0)$ refers to the $R-R$ interval average of all the centre points, $X(1)$ average of the first heartbeat interval to the right of the centre point, $X(-1)$ average of the first heartbeat interval to the left of the centre point, and $X(-2)$ average of the second heartbeat interval to the left of the centre point. Calculation formula $D C=[X(0)+X(1)-X(-1)-X(-2)] \times 1 / 4$. The unit for DC was ms.

At present, there is no explicit reference range for the normal value ofDC in the world. In this study, according to the literature, DC values were divided into three levels, namely, low risk value of sudden death ( $D C>4.5 \mathrm{~ms}$ ), moderate risk value of sudden death $(2.5 \mathrm{~ms}){ }^{4}$

SPSS 25.0 statistical software was applied for statistical analysis. Enumeration data were expressed with $n$ (\%). Measurement data were expressed with mean \pm standard deviation. The Chi-square test was employed to compare the proportion of DC values of Group A and Group B in different levels. Statistical significance was set at $p<0.05$.

In group A, there were 42 male cases (51.81\%) and 40 female cases $(48.19 \%)$, with the age of $33-76(58.37 \pm 2.15)$ years. The course of diabetes range from one month 15 years, with average course of $7.85 \pm 1.03$ years. In group $B$, there were 33 male cases $(50.77 \%)$ and 32 female cases $(49.23 \%)$, with the age of $32-75(58.06 \pm 2.31)$ years.

The proportion of DC values in high risk values of sudden death was $9.64 \%$ (8 cases) in group $A$, higher than that in group $B(0, p$ $=0.010$ ). The proportion of $D C$ values in moderate risk values of sudden death was $42.17 \%$ (35 cases) in group A, higher than that in group $B(7.69 \%, 5$ cases, $p<0.001)$. The proportion of DC values in low risk values of sudden death was $48.19 \%$ (40 cases) in group A, higher than that in group B $(92.31 \%, 60$ cases). The comparison of both groups had statistical significance ( $p$ $<0.001)$.

Vagus is the decelerating nerve of the heart. When DC declines, vagus excitability lowers, and the protection function for human body decreases. Thus, the risk of sudden death increases. This study found that the proportions of DC values of patients with type 2 diabetics in Group A were far higher than those of the normal people in Group B in high risk values and moderate risk values of sudden death, indicating that the vagus deceleration capacity of patients with type 2 diabetics weakens, compared with the normal people. The autonomic nervous function was damaged seriously, suggesting the increase of sudden death risk in the cardiovascular events. Thus, detecting DC value of patients with type 2 diabetics can well assess their autonomic 
nervous functions. This research result was consistent with the previous study. ${ }^{5}$

Detecting DC value can screen the people with seriously impaired autonomic nervous functions among the patients with type 2 diabetics. Discovering the high risk people of sudden death contributes to clinical early prevention and treatment, and reduces or avoids the serious consequences of autonomic neuropathy. The promotion of DC detection will bring the farreaching influence on prevention and treatment of sudden cardiac death for the patients with type 2 diabetics.

\section{CONFLICT OF INTEREST:}

Authors declared no conflict of interest.

\section{AUTHORS' CONTRIBUTION:}

XW: Study concept, data collection, data analysis and writing.

CZ: Data analysis.

JZ: Data collection.

QS: Study concept, critical review and revision, and final approval of the article.

\section{REFERENCES}

1. Nagai Y, Mukai K, Otsuki M, Kimura T, Kozawa J, Nishizawa $\mathrm{H}$, et al. Suppression failure of cortisol secretion by dexamethasone may occur in glucagon-like peptide-1 receptor agonist-treated patients with diabetic autonomic neuropathy. Intern Med 2018; 58(7):949-53.

2. Sztanek F, Jebelovszki É, Gaszner B, Zrínyi M, Páll D, Kempler $\mathrm{P}$, et al. Diagnosis of diabetic cardiac autonomic neuropathy. ORV Hetil 2019; 160(35):1366-75.
3. Arsenos P, Manis $G$, Gatzoulis KA, Dilaveris $P$, Gialernios $T$, Angelis $A$, et al. Deceleration capacity of heart rate predicts arrhythmic and total mortality in heart failure patients. Ann Noninvasive Electrocardiol 2016; 21(5):508-18.

4. Bauer A, Kantelhardt JW, Barthel P, Schneider R, Mäkikallio $\mathrm{T}$, UIm K, et al. Deceleration capacity of heart rate as a predictor of mortality after myocardial infarction: Cohort study. Lancet 2006; 367(9523):1674-81.

5. Wang XD, Zhou L, Zhu CY, Chen B, Chen Z, Wei L. Autonomic function as indicated by heart rate deceleration capacity and deceleration runs in type 2 diabetes patients with or without essential hypertension. Clin Interv Aging 2018; 13:1169-76.

Xisong Wang ${ }^{1}$, Chunlai Zhang ${ }^{2}$, Jiabing Zhang ${ }^{3}$ and Qing Song ${ }^{1}$

${ }^{1}$ Critical Care Medicine, Chinese PLA General Hospital, PLA Medical College, China

${ }^{2}$ Department of Cardiology, Tangshan Workers' Hospital, China

${ }^{3}$ Graduate College, Chinese PLA General Hospital, PLA Medical College, China

Correspondence to: Qing Song, Critical Care Medicine, Chinese PLA General Hospital, PLA Medical College, 100853, China

E-mail: pqsy2uk@163.com

Received: October 10, 2019; Revised: December 21, 2019; Accepted: January 24, 2020

DOI: https://doi.org/10.29271/jcpsp.2020.09.992 